
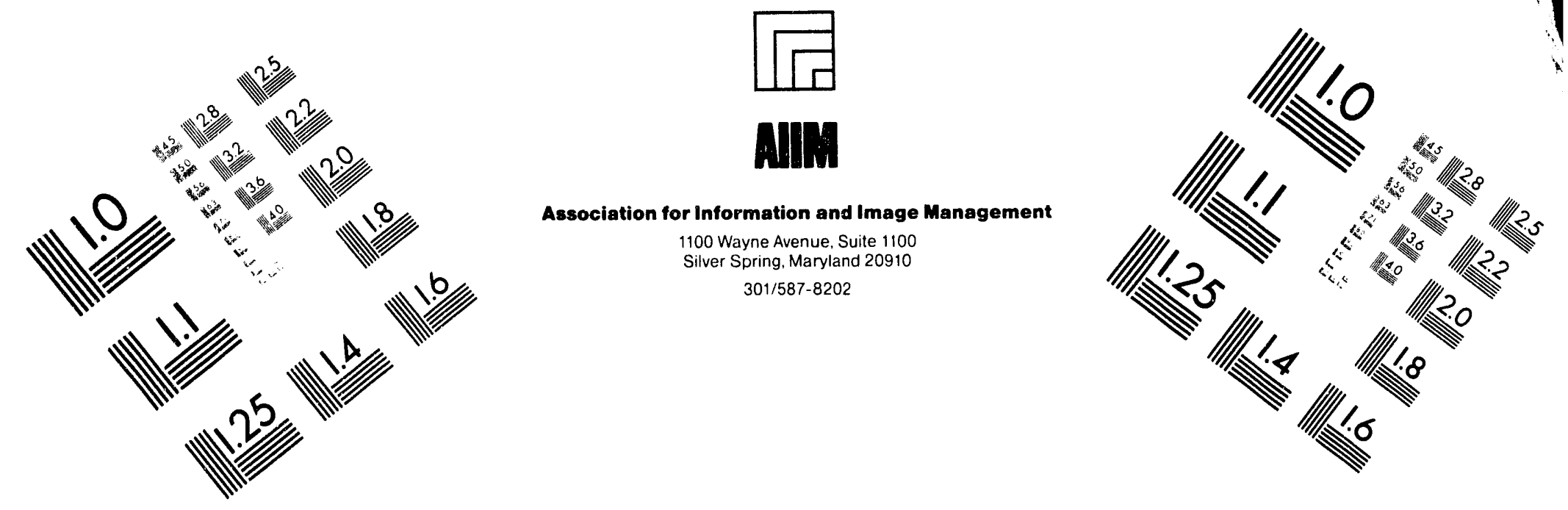

Centimeter

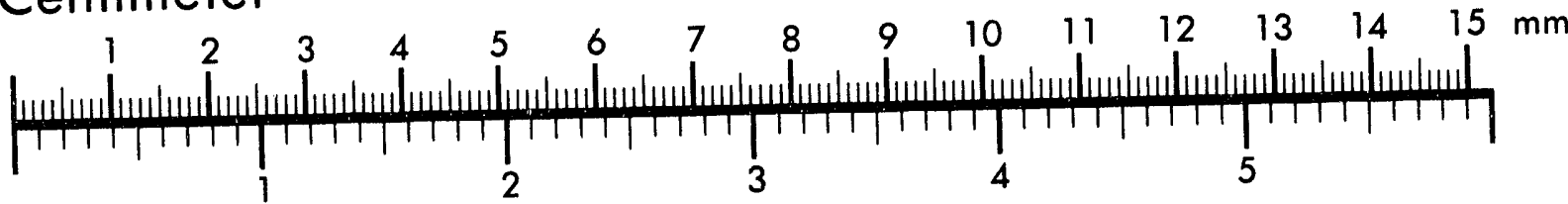
Inches
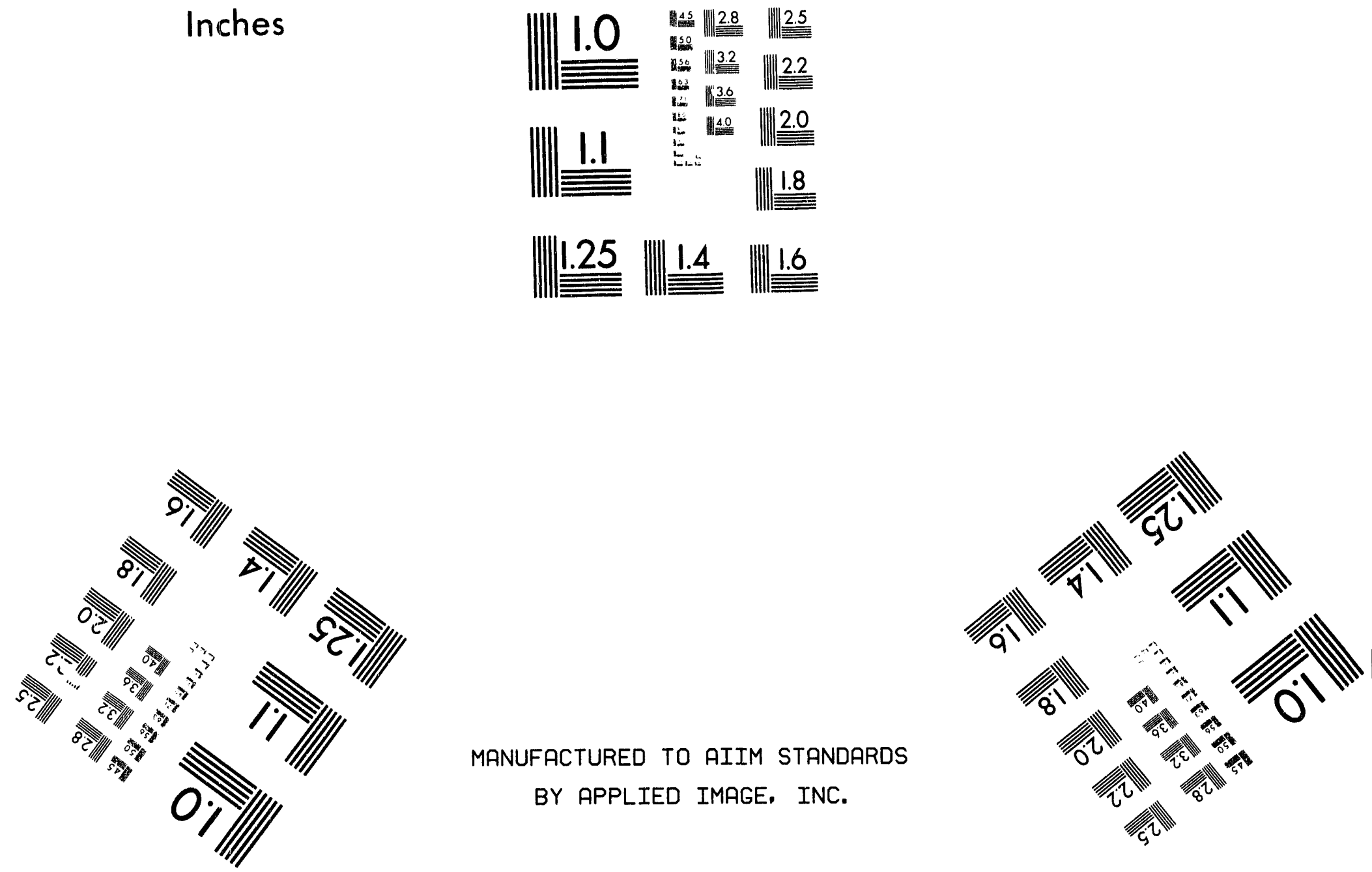

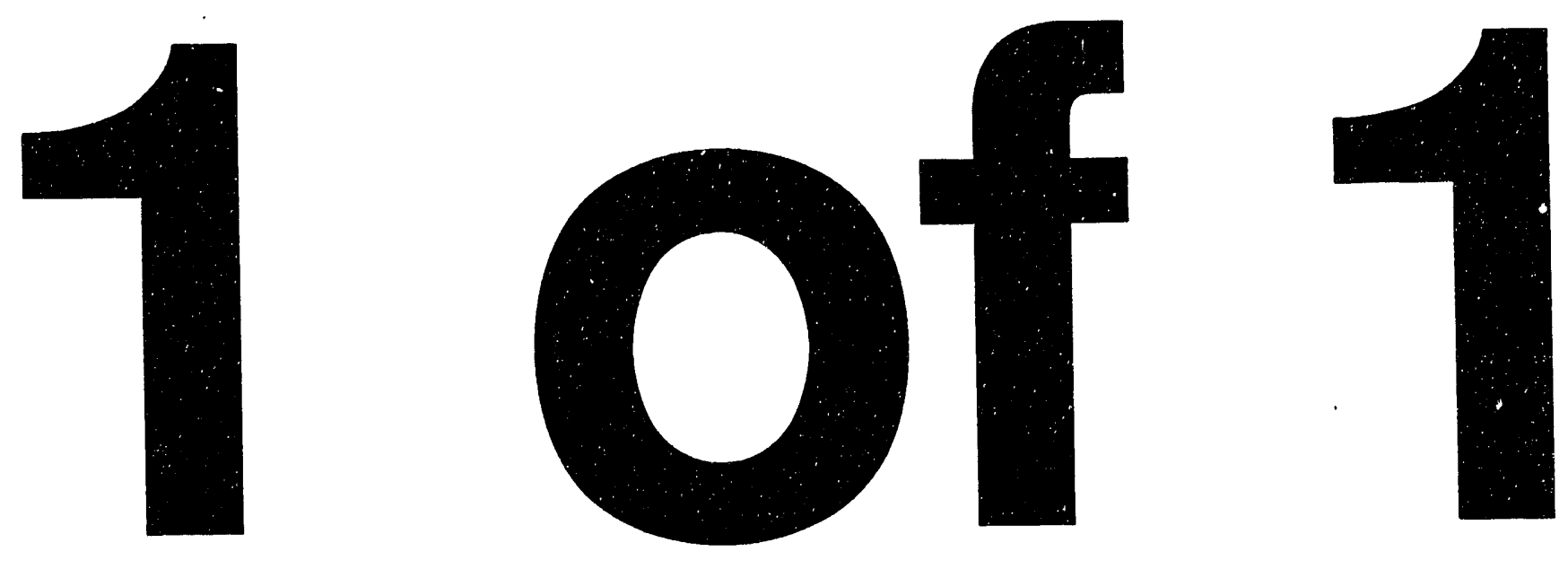


\title{
Predicting the Vibrations of a Spinning Inflated Membrane ${ }^{1}$
}

\author{
Daniel J. Segalman \\ Department 1434 \\ and \\ Adam Slavin \\ Department 1562 \\ Sandia National Laboratories \\ Albuquerque, NM 87185
}

\subsection{Introduction}

The primary difficulty of computing the vibration of spinning inflated membranes arises from the low natural frequencies of such systems. When such systems are rotated near their own natural frequencies the dynamics equations must account for higher order kinematics than is necessary for more rigid structures. These complications results from the membrane loads that develop within the bodies in reaction to the accelerations of the overall body. When second order kinematics act against these membrane loads, the resulting energies become of the same order as the potential and kinetic energies of the vibrations that would be calculated by first order kinematics.

These complications apply to the problem addressed here. Here we consider a spin-stabilized, inflated membrane, spinning around its minor axis. This structure is very flexible and somewhat viscoelastic, so vibrations excited by the overall motion of the structure will dissipate energy of the system, thus reducing the kinetic energy. A reduction in kinetic energy consistent with a conservation of angular momentum results in coning and, eventually, tumbling. Here we must address the excitation of vibration by the rigid-body motion and then we must address the retarding effect of the energy dissipation on the rigid-body motion.

The difficulties of the dynamics of flexible systerns rotating near their first natural frequencies has been addressed frequently in the literacure recently. The literature is too large to reference fully, but several much discussed monographs are References [1],[2], and [3]. One traditional approach to addressing these difficulties is to uncouple the vibratory motion of the structure from the "rigid-body" motion except for an a-posteriori aldition of

1. Work supporied by the U.S. Department of Energy at Sandia National Lahoratorics under Contract DEAC(0476DP()()789

Predicting the Vihrations of a Spinning Inflated Membrane 
"spin-stiffening". Though this approach is very often successful, it cannot result in the desired prediction of the secular effect of vibratory dissipation on the overall motion. Another, though brute-force, approach involves the recalculation of the stiffness matrix and inertial loads at each time step. Though theoretically acceptable, that approach proved to be prohibitively slow and prohibitively expensive.

\subsection{Analysis Technique}

Here we report the application of one method that was developed for addressing flexible rotating systems to the calculation of the dynamics of an inflated membrane. This method, presented in Reference [4], involves the use of a space of accessible configurations that are consistent with the nonlinear static properties of the structure up to second order in the applied force.

Beginning with kinematics, a particle originally at location $\chi$ will occupy a position $\boldsymbol{x}(\chi, t)$ at time $\mathrm{t}$ :

$$
\boldsymbol{x}(\chi, t)=p(t)+R(t) \cdot[\chi+U(\chi, t)]
$$

where the rotation tensor $R(t)$ accounts for rigid-body rotation and the vector $\boldsymbol{p}(t)$ accounts for rigid-body translation. All remaining displacement is absorbed in to the $U(\chi, t)$ term. There is some ambiguity in the distribution of displacements among $R(t)$, $p(t)$, and $\boldsymbol{U}(\chi, t)$ until one adds two more vector constraints. We have chosen to require that $\boldsymbol{p}(t)$ move as the instantaneous center of gravity of the deforming structure and that $R(t)$ rotate along with the instantaneous moment of inertia tensor.

A representati on for the deformation field, $U(\chi, t)$, is obtained in a manner described below. One devises an expansion for the nonlinear static response of the structure that is correct up to second order in the imposed forces for a basis of force fields:

$$
\begin{gathered}
\boldsymbol{F}(\chi, t)=\sum_{m} s_{m}(t) \boldsymbol{f}^{m}(\chi) \\
\boldsymbol{U}(\chi, t)=\sum_{m} s_{m}(t) \boldsymbol{u}^{m}(\chi)+\sum_{m, n} s_{m}(t) s_{n}(t) \boldsymbol{g}^{m, n}(\chi)
\end{gathered}
$$

The imposed force field $\boldsymbol{F}(\chi, t)$ has been expressed as a series of basis fields $f^{m}(\chi)$ and the displacement field has been expressed as a series of linear and quadratic terms, the identity of each must be deduced from the solution of the nonlinear statics. Note that the linear and quadratic fields, $\boldsymbol{u}^{m}(\chi)$ and $g^{m, n}(\chi)$, are each derived from the force basis.

These kinematics of Equation 1 and Equation 3 are employed with Hamilton's principle in the derivation of the governing equations of the system. Even after the governing equations are derived and linearized with respect to the deformation parameter $s_{m}(t)$, there exist terms lirear in the fields $g^{m, n}(\chi)$, reflecting the work done against the second order deformations. 
The derivation and governing equations are given in Reference [4]. Also in that reference are several very simple illustrations of the method. The governing equations involve several matrices of constant integrals taken over the body, akin to mass and stiffness matrices, that must be calculated prior to performing time integration. For the simple problems of Reference [4], those integrals were calculated in a direct manner. For the complex structure presented here, it was necessary to develop extensive computer code to perform the integrals accounting for the kinematics of the full structure. The development and nature of that code will be a topic of another paper and will be discussed in only a cursory manner here.

When Hamilton's principle is used with the above kinematics, after an integration by parts, and after numerous tensor identities have been exploited, the following governing equations result for the linear acceleration, $\ddot{p}(t)$, for the angular acceleration $\dot{\Omega}$, and for the second derivative, $\ddot{s}_{m}(t)$, of the deformation coordinate:

$$
\begin{gathered}
0=\int_{\text {volume }}\left[-\rho(\chi) \ddot{\boldsymbol{x}}(\chi, t)+\boldsymbol{F}_{X}(\chi, t)\right] d V \\
0=\int_{\text {volume }}\left\{R(t) \cdot\left[\chi+\sum_{m} s_{m} \boldsymbol{u}^{m}\right]\right\} \times\left[-\rho(\chi) \ddot{\boldsymbol{x}}(\chi, t)+\boldsymbol{F}_{X}(\chi, t)\right] d V
\end{gathered}
$$

and

$$
0=\int_{\text {volume }}\left\{R(t) \cdot\left[\boldsymbol{u}^{m}+2 \sum_{n} s_{n} g^{m, n}\right]\right\} \cdot\left[-\rho(\chi) \ddot{\boldsymbol{x}}(\chi, t)+\boldsymbol{F}_{X}(\chi, t)-\boldsymbol{F}_{s}(\chi, t)\right] d V
$$

where $\rho(\chi)$ is local mass density; $\boldsymbol{F}_{X}(\chi, t)$ is the field of externally applied forces; and

$$
\boldsymbol{F}_{s}(\chi, t)=\sum_{k} s_{k}(t) f^{k}(\chi)
$$

is the field of reaction forces associated with the linear deformation field $\sum_{m} s_{m} u^{m}$.

The acceleration terms $\ddot{\boldsymbol{x}}(\chi, t)$ in Equation 4, Equation 5, and Equation 6 must be expressed in terms of its parts:

$$
\ddot{\boldsymbol{x}}(\chi, t)=\ddot{p}+\left(\dot{\Omega}+\Omega^{2}\right) \bullet\left[\chi+\sum_{m} s_{m} \boldsymbol{u}^{m}\right]+2 \Omega \bullet \sum_{m} \dot{s}_{m} \boldsymbol{u}^{m}+\sum_{m} \ddot{s}_{m} \boldsymbol{u}^{m}
$$

One notes that the quantities $s_{m}(t), p(t)$, and $R(t)$, and their lerivatives can be factored out of the volume integrals. All remaining terms in the integrals with the exception of $F_{X}(\chi, t)$ are independent of time. With the exception of the terms involving $F_{X}(\chi, t)$, all integrals can be evaluated once, and stored for use in the time integration problem. (Though there are methods of circumventing the difficulties of the external force terms, such terms do not occur in this problem and are not discussed further.) At each time step, one needs solve only the small system of equations for $\ddot{p}(t), \dot{\Omega}$, and $\ddot{s}_{m}(t)$, without reference to many nodal degrees of freedom. 
The volume integrals of Equation 4, Equation 5, and Equation 6 are evaluated through an unconventional use of a finite element code. One uses a nonlinear statics code in the manner suggested in Reference [4], to find expressions for $f^{m}(\chi), g^{m, n}(\chi)$, and $\boldsymbol{u}^{m}(\chi)$ in terms of nodal displacements. A dynamics code is used to extract the necessary inertial terms.

It should be noted that the total number of scalar equations in the above system is $6+\mathrm{N}$, where $\mathrm{N}$ is the number of deformation modes considered. Therefore, calculation at each time step involves only a small number of degrees of freedom and does not require any additional spacial integrals at that step.

Examination of Equation 6 provides some insight as to the rigid body motions that will excite vibrational response. Inner products of the sort

$$
\int_{\text {volume }}\left\{R(t) \cdot\left[\boldsymbol{u}^{m}+2 \sum_{n} s_{n} \boldsymbol{g}^{m, n}\right]\right\} \cdot[-\rho(\chi) \ddot{\boldsymbol{x}}(\chi, t)] d V
$$

represent loadings on the vibration modes by acceleration terms including those involving $\Omega$ and its derivatives. When the rotational rate is on the order of a natural frequency, one would expect some excitement of resonance.

\subsection{The Inflated Membrane}

The mesh used to model the structure addressed here is shown in Figure 1 and Figure 2.

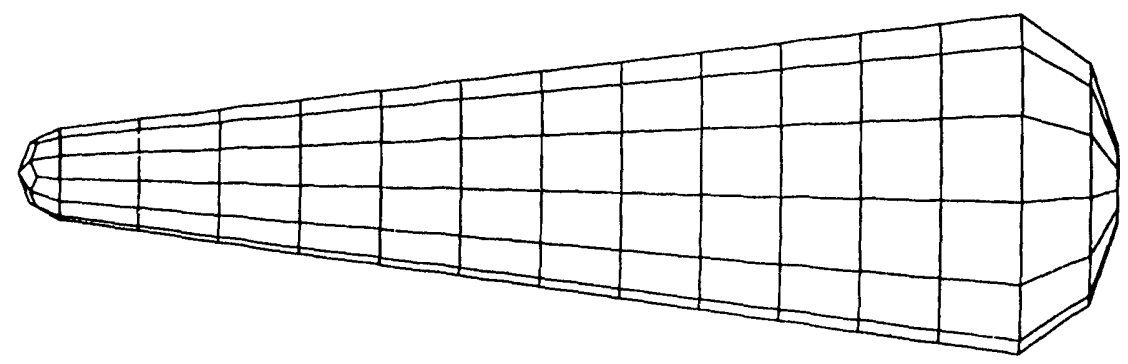

FICURE 1. A side view of the mesh of the modelled structure. 


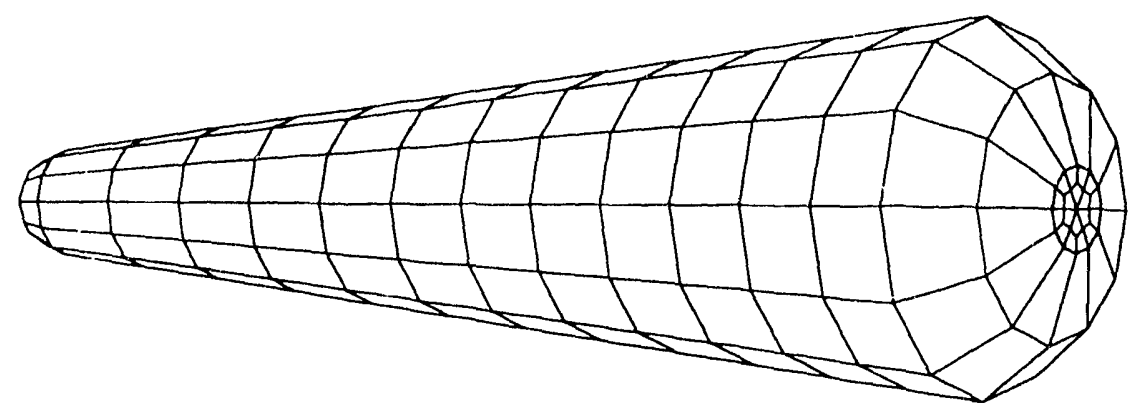

FIGURE 2. A rotated view of the mesh of the modelled structure.

The structure is approximately conical in shape and approximately 60 inches long with a nearly spherical cap of approximately 5 inch diameter on the front and a nearly spherical cap of approximately 20 inch diameter on the back. There are also small masses affixed to the front and rear of the structure. The properties of the membrane material are listed in Table 1, "Membrane Material Properties," on page 5.

Two cases were run, involving:

- an initial spin rate of $0.5 \mathrm{~Hz}$

- an initial spin rate of $41 \mathrm{~Hz}$.

In each case the membrane was inflated to 0.3 psi so that the first frequency was $39 \mathrm{~Hz}$; there was an initial coning angle of approximately $6^{\circ}$; and Euler backward integration was performed every 2.5 milliseconds. Though the exact meaning of "coning angle" in a deforming structure is unclear, the initial conditions were specified in terms of the orientation of the instantaneous principle axis vectors.

The analysis was performed using basis fields $f^{\prime \prime}(\chi)$ corresponding to the first five eigen modes of the sistem. (These were evaluated formally by contracting the eigenmodes with the mass matrix.) The linear deformations, $\boldsymbol{u}^{m}(\chi)$, are the eigen modes, and the coefficients, $g^{m, n}(\chi)$, of the quadratic terms are calculated from the nonlinear statics in the manner outlined above.

Table 1: Membrane Material Properties

\begin{tabular}{|c|c|}
\hline Material Property & Value \\
\hline Density & $0.0335 \mathrm{lb} / \mathrm{in}^{3}$ \\
\hline Elastic Modulus & $0.05 \mathrm{Mpsi}$ \\
\hline
\end{tabular}




\section{Table 1: Membrane Material Properties}

\begin{tabular}{|c|c|}
\hline Material Property & Value \\
\hline Poisson's Ratio & 0.3 \\
\hline Thickness & 0.0045 in \\
\hline \%Critical Damping & 2.0 \\
\hline
\end{tabular}

The deformation modes are illustrated in Figure 3, Figure 4, and Figure 5. These were selected on the reasoning that the overall deformation only introduces significant excitation forces to the system whose frequencies are at or below the spin rate of the system, so the modes associated with frequencies at and below that value should be sufficient.

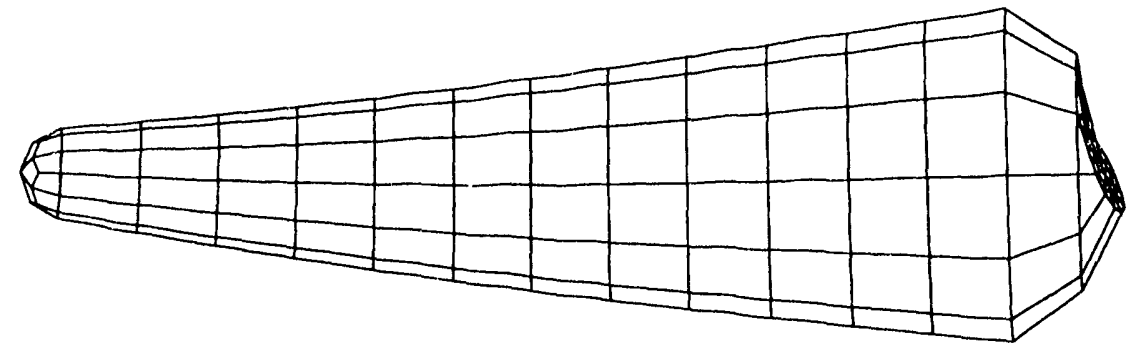

FIGURE 3. The first and second deformational modes involve primarily a rocking of the mass at the base of the balloon. These occur at $31.2 \mathrm{~Hz}$.

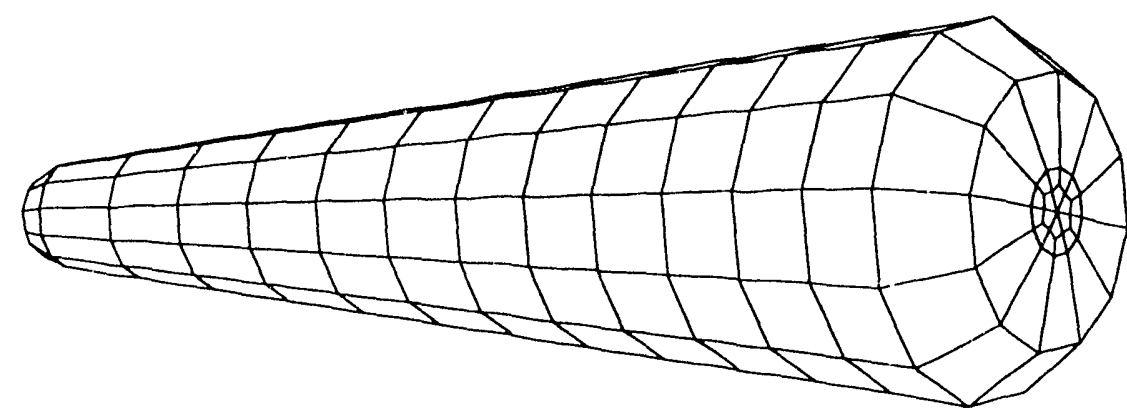

FIGURE 4. The third mode is an in-and-out motion of the rear mass. 


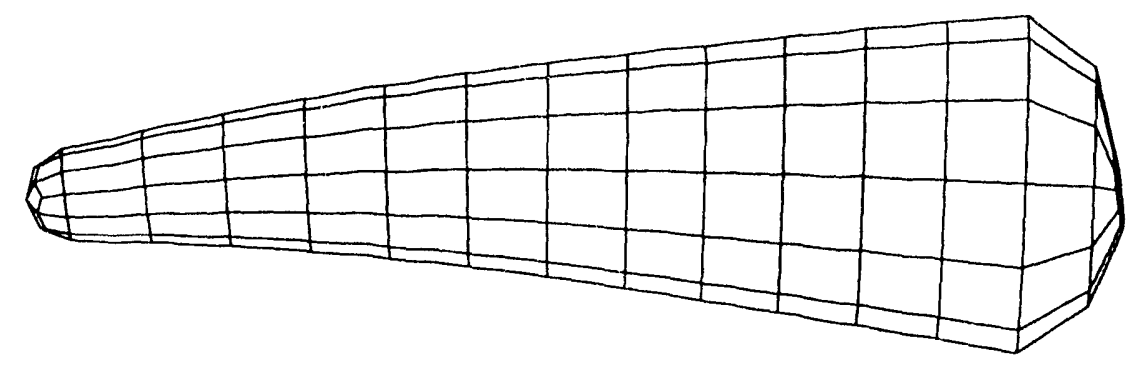

FIGURE 5. The fourth and fifth modes are bending modes of the overall structure. These occur at $38.0 \mathrm{~Hz}$.

\subsection{Results}

As expected, since the spin rate of the first problem was well below the first natural frequency of the structure, there was very little coupling between the rigid-body part of the motion and the vibratory motion. An examination of Figure 6 shows that, as expected, the magnitude of the anguiar momentum vector is conserved. Though not show here, the orientation of that vector is also conserved. It is in Figure 7 where the physics of this problem becomes interesting. A deformation introduced as an initial condition to this problem dies out with a log-decrement consistent with $2 \%$ damping. The small decline in total vehicle energy is evidenced in Figure 8. The spin rate and cone angle are shown to be nearly constant in Figure 9 and Figure 10, as expected. (Despite the absence of resonance there may still be a very slow energy leakage due to forced, nonresonant vibration.)

The situation is somewhat different for the case of an initial spin rate of $41 \mathrm{~Hz}$. Though the momentum is conserved, as evidenced in Figure 11, there is substantial interaction between the vibrational modes and the rigid-body motion. Figure 12 shows the coefficient of the first bending mode. At first there is a beating-like phenomena reflecting the interaction between several modes. (Note the $g^{m, n}(\chi)$ terms in Equation 6 destroy orthogonality of modes). Eventually there is a steady growth in the vibration magnitude, indicating a flow of energy from the rigid-body rotation.

The energy dissipation associated with the growing vibrations results in a decay of system energy, as shown in Figure 14. This lost energy comes primarily from the spin rate, as shown in Figure 15. Conservation of angular momentum in the presence of decreasing spin rate requires an increased moment of inertia bout the axis parallel to the coning axis a condition satisfied by an increasing coning angle. The evolution of the cone angle is show in Figure 16.

It should be noted that the early-time oscillations in total energy are of very small amplitude $(\sim 0.1 \%$ of the mean value) and that these oscillations dissappear when the early-time calculations are rerun with much smaller time step. 


\section{Momentum Magnitude}

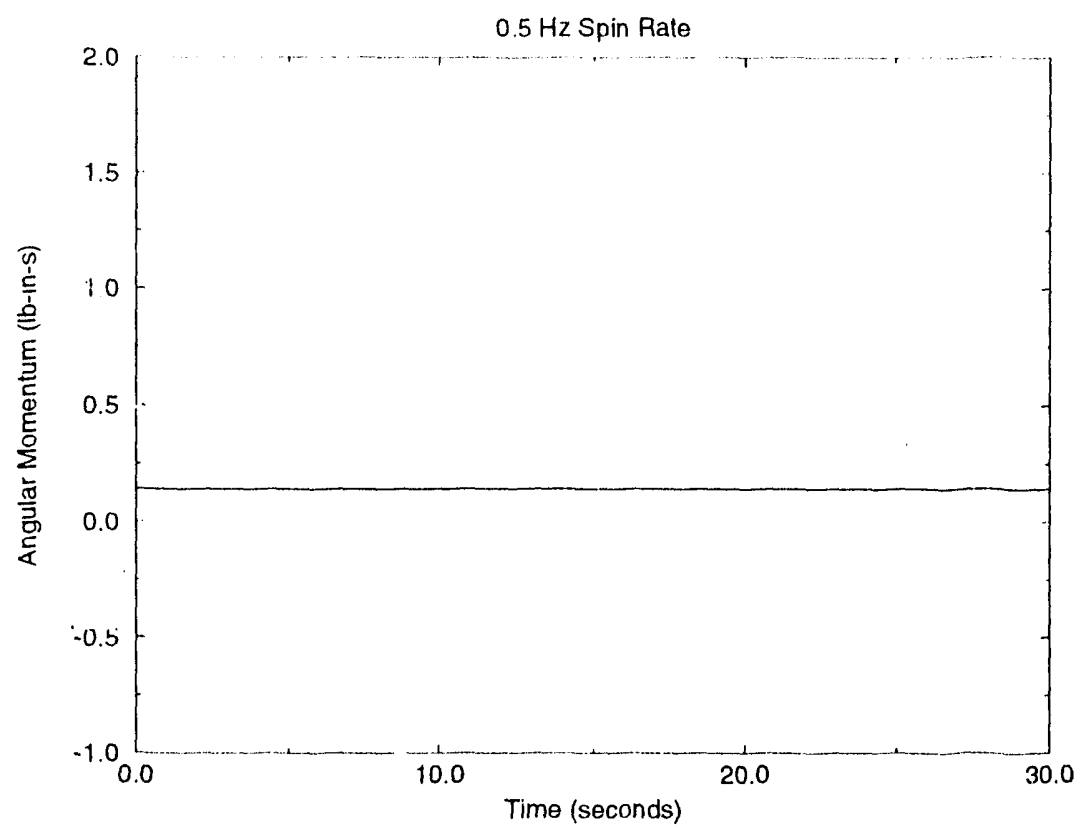

FIGURE 6. The magnitude of the momentum vector as a function of time for an initial spin rate of $0.5 \mathrm{~Hz}$.

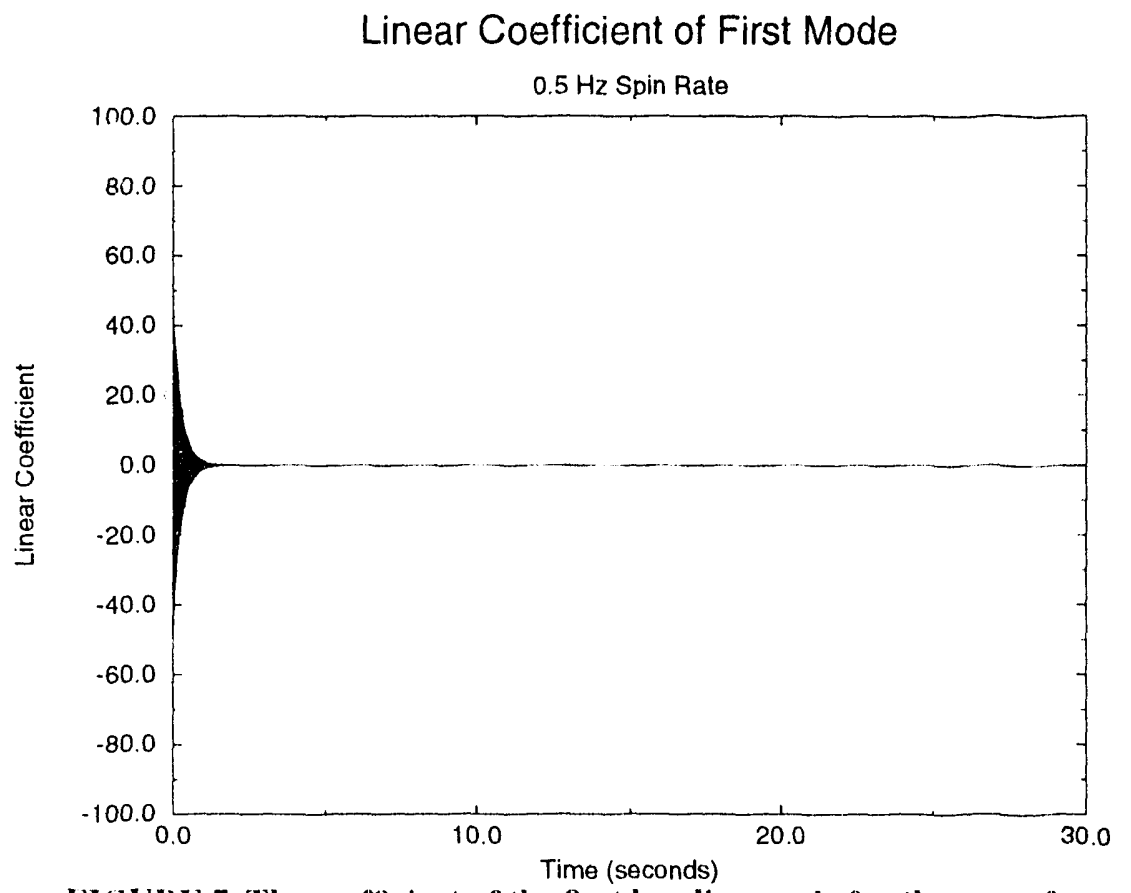

FICURE 7. The coeficient of the first bending mode for the case of an initial spin rate of $0.5 \mathrm{~Hz}$. The initial deformation quickly decays to zero, without apparent excitation from the rigid body motion. 


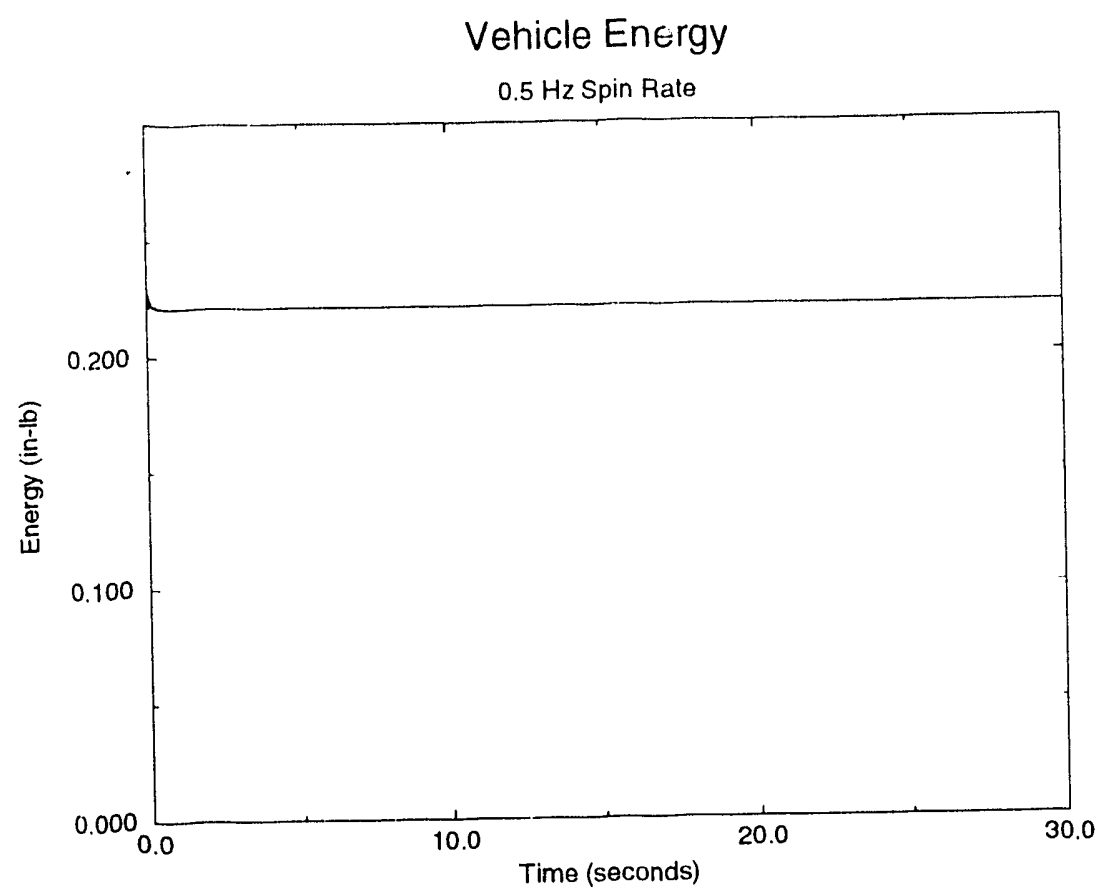

FIGURE 8. Total kinetic and potential energy for the case of an initial 0.5 Hz spin rate.

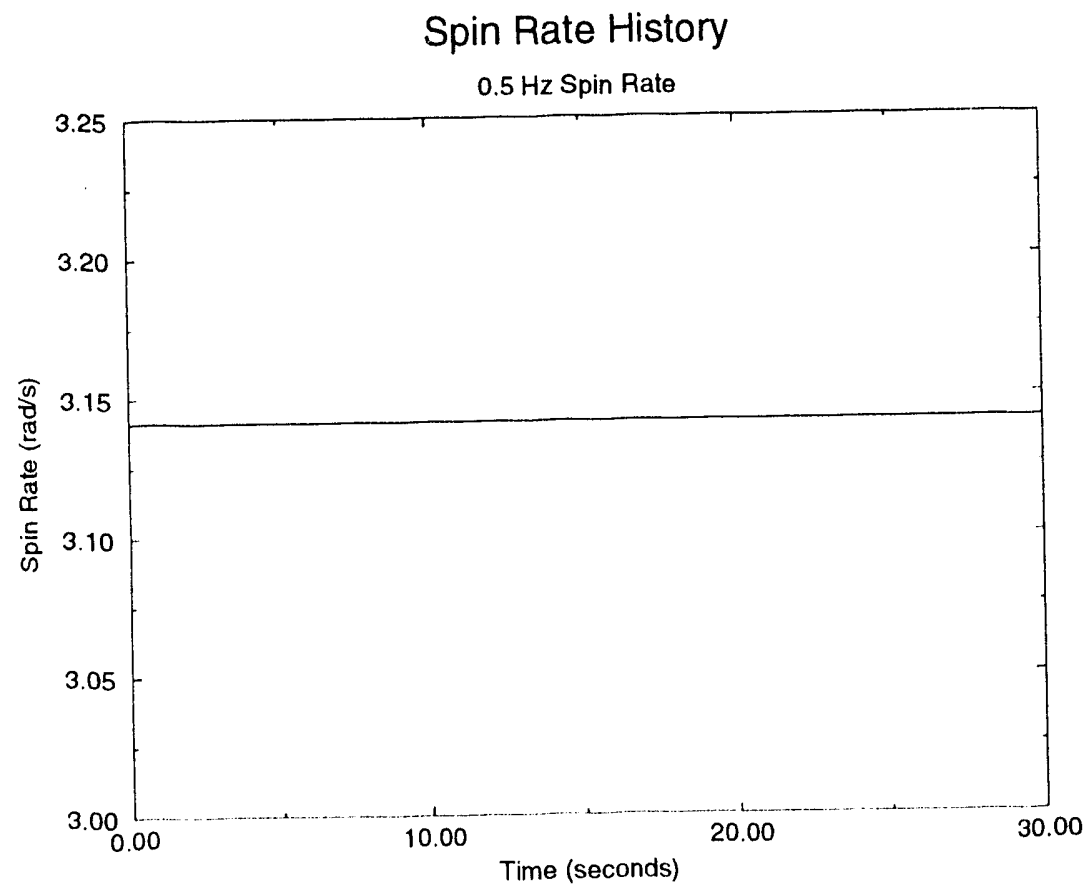

FI(GURE 9. Spin-rate history for the case of an initial $0.5 \mathrm{~Hz}$ spin rate. Note that this behavior is consistent with that of a rigid body. 


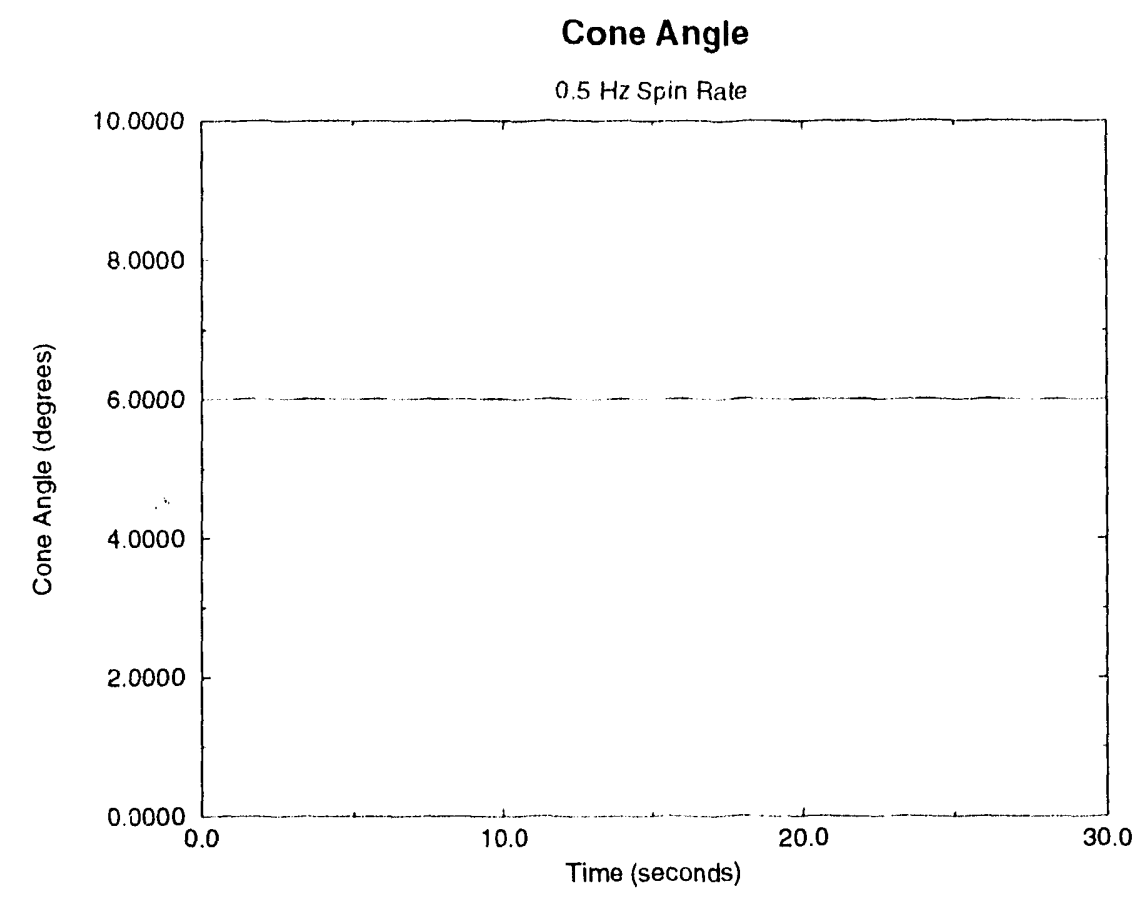

FIGURE 10. The magnitude of the cone angle as a function of time for an initial spin rate of $0.5 \mathrm{~Hz}$.

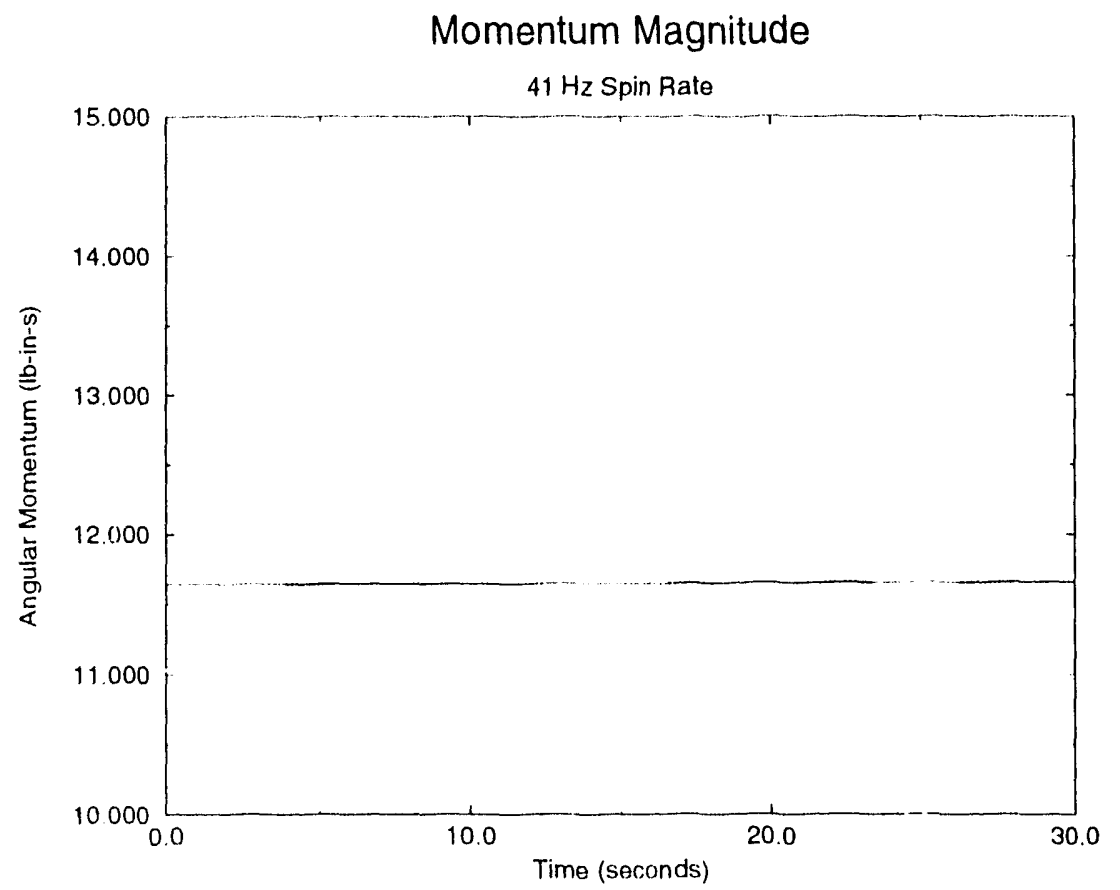

FIC:URE 11 . The magnitude of the angular momentum vector for the case of an initial spin rate of $41 \mathrm{~Hz}$. This plot demonstrates the conservation of angular momentum. 
Linear Coefficient of First Mode

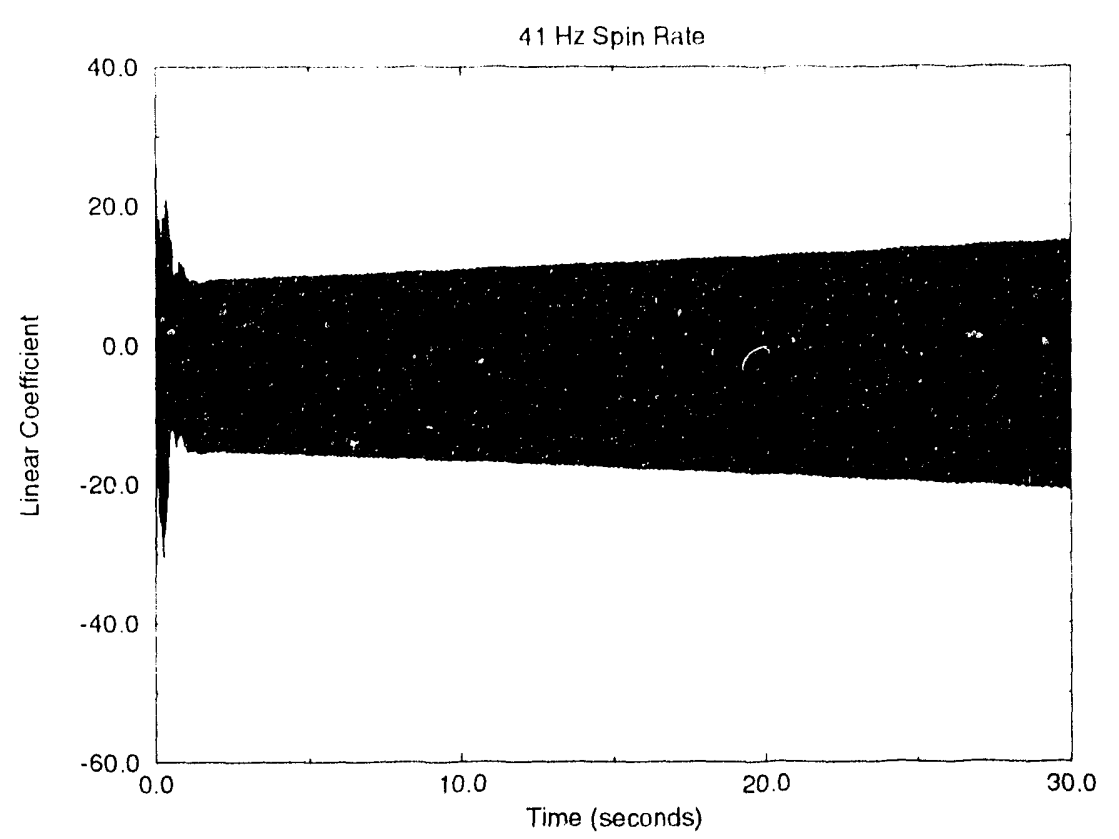

FIGURE 12. The coefficient of the first bending mode for that case of an initial spin rate of $41 \mathrm{~Hz}$.Vibratory dissipation removes kinetic energy from the system.

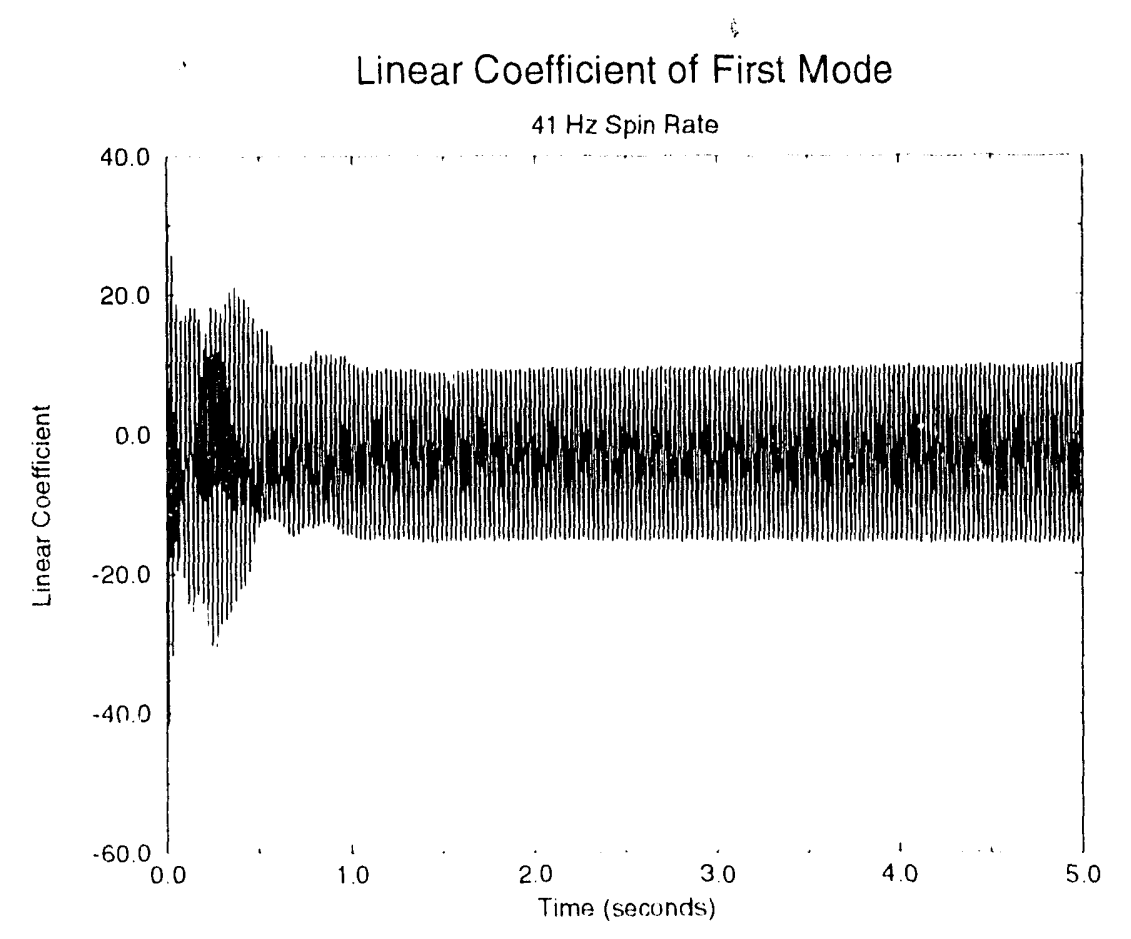

FICURL: 13. The coefficient of the first bending mode for that case of an initial spin rate of $41 \mathrm{~Hz}$ - a magnified view at early times. 


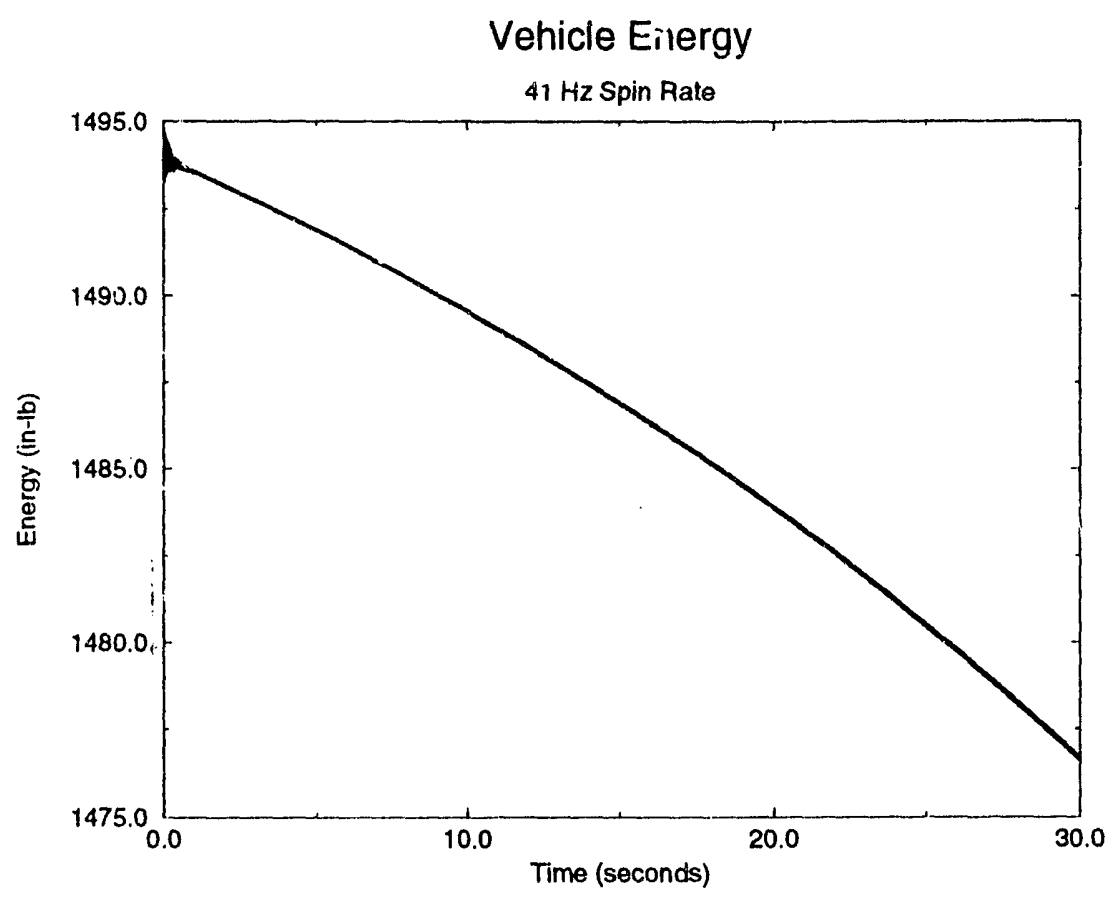

FIGURE 14. The total energy for the case of an initial spin rate of $41 \mathrm{~Hz}$. Vibratory dissipation removes kinetic energy from the system.

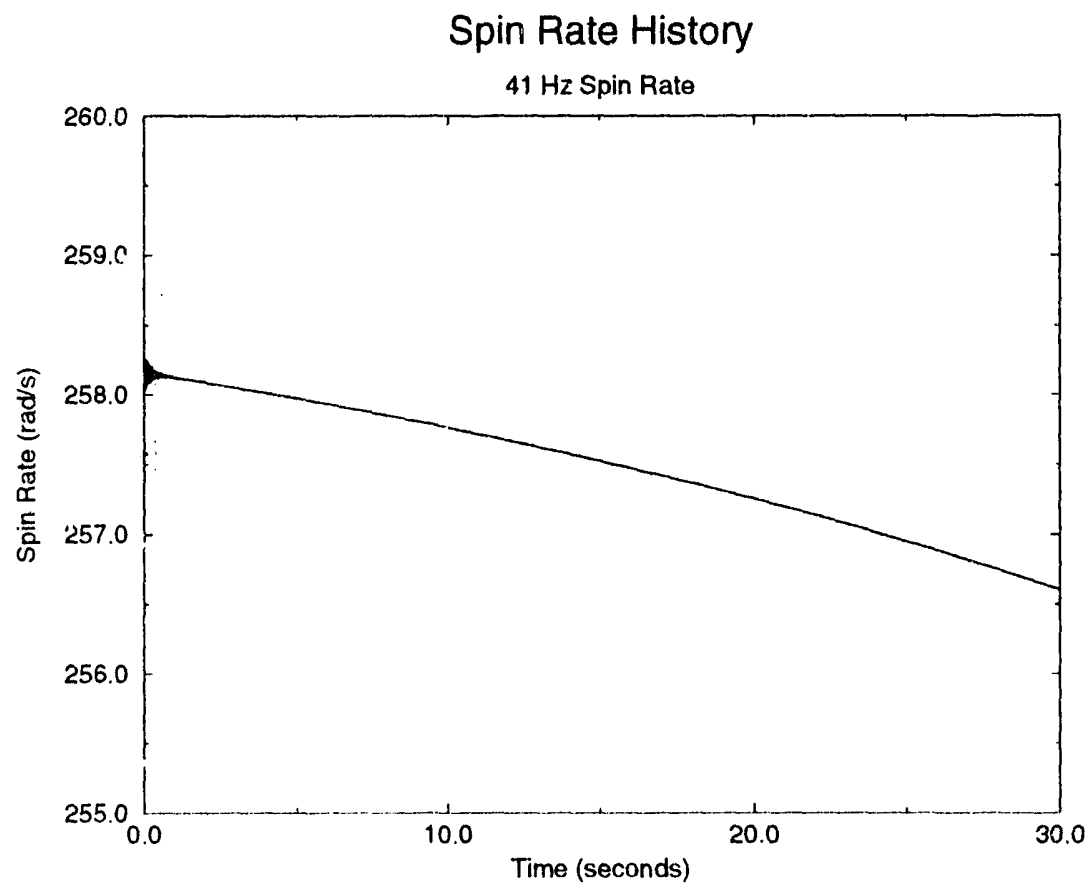

FIGURE 15. Spin rate history for the case of an initial spin rate of $41 \mathrm{~Hz}$. Vibratory dissipation removes kinetic energy from the system. 


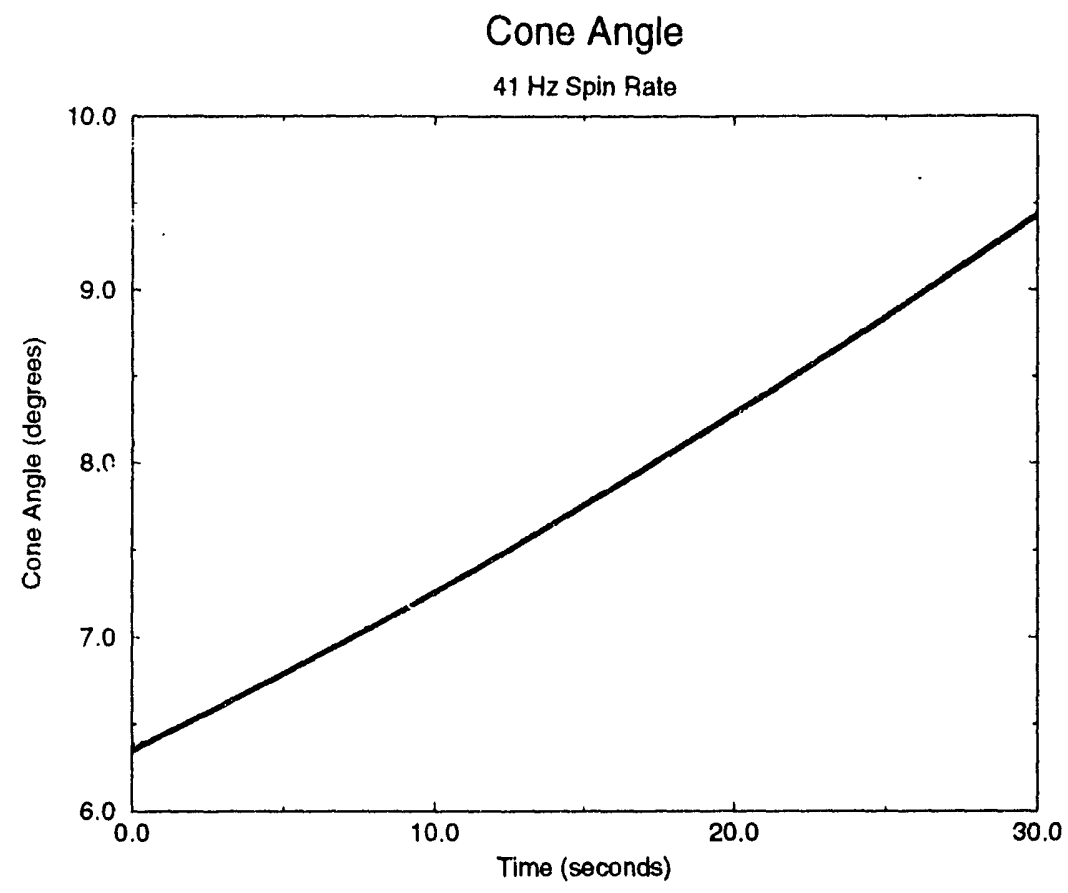

FIGURE 16. The cone angle for the case of an initial spin rate of $41 \mathrm{~Hz}$. Note that the cone angle increases with time as energy leaves the system. 


\subsection{Conclusions}

Though orthogonality of the linear modes is lost in vibration problems involving high speed rotation - that is high-speed relative to the $\mathrm{r}$ igen frequencies - such problems can still be addressed in a manner involving just a few degrees of freedom so long as all the necessary physics is accommodated.

The calculations show for this case, as argued heuristically above, that rigid body rotation will not excite vibrational modes associated with frequencies much higher than the magnitude of the spin tensor.

Most importantly, the method of quadratic modes has been demonstrated to give reasonable predictions for problems of the sort presented here. Further, since such "soft" structures as inflated membranes are especially numerically difficult, we expect this method to be similarly successful in other problems of rotation of flexible bodies.

\subsection{References}

1. Fidelis O. Eke and Robert A. Laskin, 'On the Inadequacies of Current Multi-Flexible Body Simulation Codes', Proceedings of the Guidance, Control, Navigation, and Control Conference, Monterey, CA, August 1987, pp 79-89, AlAA Paper No 87-2248

2. C. E. Padilla and A. H. von Flotow, 'Non-linear Strain-Displacement Relations and Flexible Multibody Dynamics', Proceedings of the 30'th AIAA/ASME/ASCE/AHS/ASC Structures, Structural Dynamics, and Materials Conference, Mobile Alabama, April 1989, pp $105-413$

3. T. R. Kane, R. R. Ryan, and A. K. Banerjee, 'Dynamics of a Cantilever Beam Attached to a Moving Base', AIAA J. Guidance, Control, and Dynamics, Volume 10, 1987, pp 139-151

4. Daniel J. Segalman and Clark R. Dohrmann, 'Dynamics of Rotating Flexible Structures by a Method of Quadratic Modes', Sandia Report: SAND90-2737, printed December 1990

\section{DISCLAIMER}

This report was prepared as an account of work sponsored by an agency of the United States Government. Neither the United States Government nor any agency thereof, nor any of their employees, makes any warranty, express or implied, or assumes any legal liability or responsibility for the accuracy, completeness, or usefulness of any information, apparatus, product, or process disclosed, or represents that its use would not infringe privately owned rights. Reference herein to any specific commercial product, process, or service by trade name, trademark, manufacturer, or otherwise does not necessarily constitute or imply its endorsement, recommendation, or favoring by the United States Government or any agency thereof. The views and opinions of authors expressed herein do not necessarily state or reflect those of the United States Government or any agency thereof. 

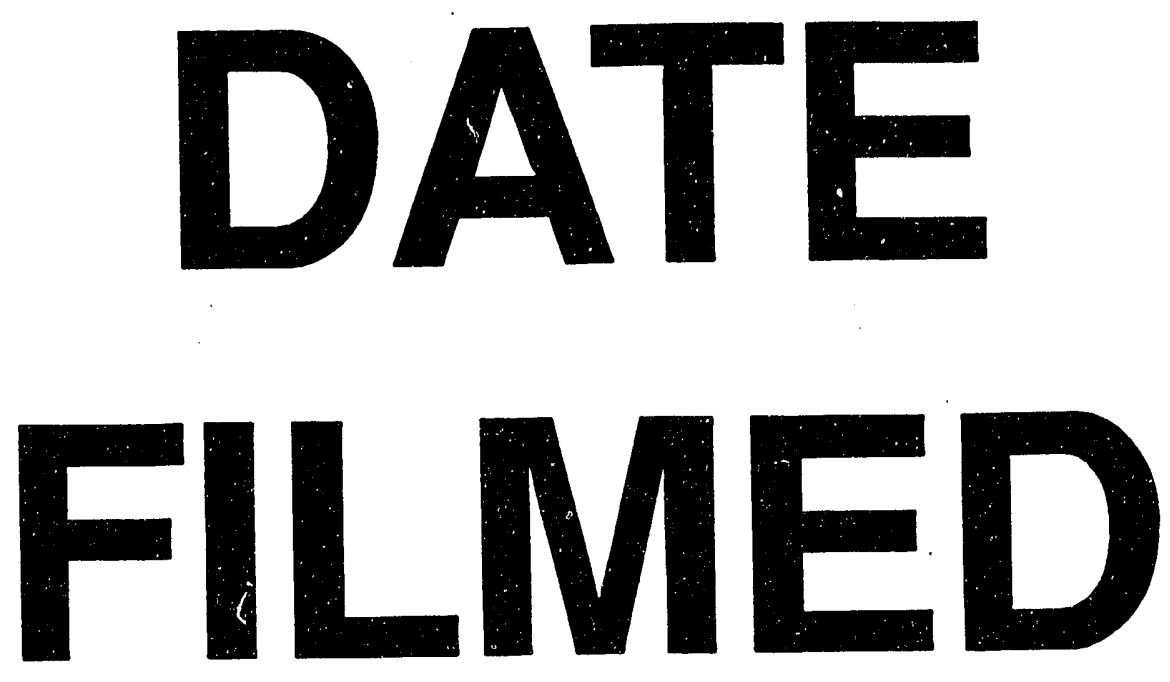

$8 / 18 / 93$
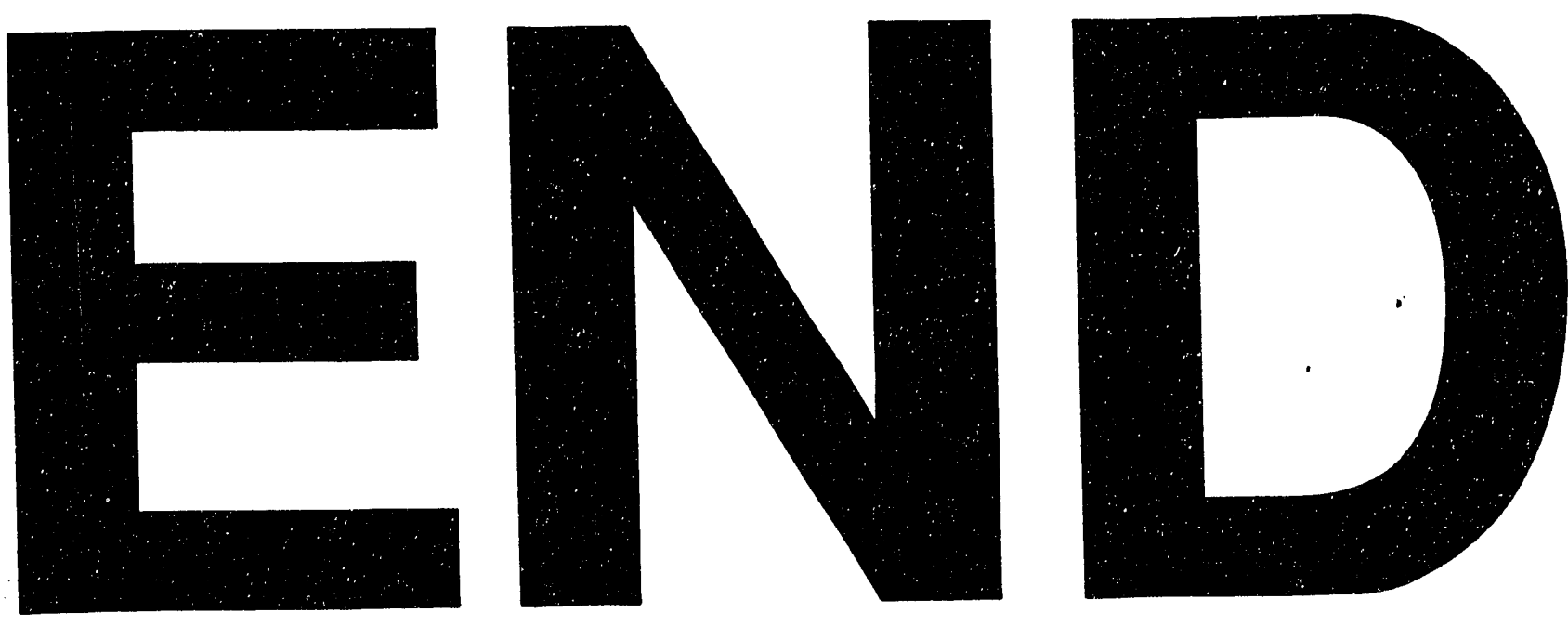
